\title{
BIOLOGIA REPRODUTIVA DE PEIXE COELHO SAPATEIRO, Siganus sutor (VALENCIENNES, 1835) EM CABO DELGADO, NORTE DE MOÇAMBIQUE
}

\section{REPRODUCTVE BIOLOGY OF WHITESPOTTED RABBITFISH, Siganus sutor (VALENCIENNES, 1835) IN CABO DELGADO, NORTHERN MOZAMBIQUE}

\author{
Sidónio Paulino Raúl MACHAIEIE ${ }^{1 *} \&$ Isabel Marques da SILVA ${ }^{1}$ \\ ${ }^{1}$ Facudade de Ciências Naturais, Universidade Lúrio, CP 958, Pemba, Cabo Delgado, Moçambique \\ *E-mail: sdmachaieie@gmail.com
}

Recebido: 31/03/2020 / Publicado: 01/03/2021

\begin{abstract}
Resumo - O peixe coelho sapateiro Siganus sutor é um dos recursos pesqueiros mais importante das pescarias artesanais em Cabo Delgado. No entanto, a legislação pesqueira em vigor não tem qualquer regulamentação da pesca desta espécie. $\mathrm{O}$ estudo da biologia reprodutiva desta espécie é importante na medida em que permite determinar medidas de conservação visando à manutenção dos estoques pesqueiros da espécie Siganus sutor. O presente estudo teve como objetivo determinar os aspetos da ecologia reprodutiva do Siganus sutor em Cabo Delgado. Foram coletados 724 indivíduos provenientes da pesca artesanal entre agosto de 2018 a julho de 2019. No laboratório procedeu-se a triagem dos dados biométricos (peso com 5 g de precisão e comprimento com precisão de 1 $\mathrm{mm}$ ) de cada indivíduo. Posteriormente, os estágios das gonadas foram observados macroscopicamente usando uma escala de desenvolvimento, de imaturo ao desovado/aspermo baseado de critérios morfológicos. A proporção sexual para o período do estudo foi de 1,5 fêmeas para cada 1 macho. A primeira maturação foi de 187,01 mm de comprimento total para fêmeas e de 201,73 mm para machos. O período com maior proporção de peixes com gonadas maturas foi de abril a agosto. Perante este cenário, para manutenção das populações desta espécie, são sugeridas medidas de gestão que garantem a reprodução da espécie durante os meses de abril a agosto, a definição do tamanho mínimo de captura de 202 mm para fêmeas e machos.
\end{abstract}

Palavras-chave: Siganus sutor; reprodução; pesca artesanal; oceano índico ocidental; gestão pesqueira.

\begin{abstract}
Whitespotted rabbitfish Siganus sutor is one of the most important fishing resources in artisanal fisheries in Cabo Delgado. However, the fishing legislation in force does not have any fishing regulations for this species. The study of the reproductive biology of this species is important because it allows the determination of conservation measures aiming at maintaining the fish stocks of Siganus sutor. The present study aimed to determine the reproduction aspects of the Siganus sutor in Cabo Delgado. 724 individuals from artisanal fisheries were collected between august 2018 and july 2019. In the laboratory, the biometric data (weight - $g$ and length $\mathrm{mm}$ ) of individuals were screened, then the gonad stages were observed macroscopically using a development scale, from immature to spent based on morphological criteria. The sex ratio for the study period was 1.5 females for each male. The first sexual maturation was determined at a total length of 187.01 $\mathrm{mm}$ for females and $201.73 \mathrm{~mm}$ for males. The period with the most mature gonads was from April to august. Because of this scenario, for maintaining populations of this species, management measures suggested are establishing the reproduction period of the species during april to august, the definition of the minimum catch size of $202 \mathrm{~mm}$ for females and males.
\end{abstract}

Keywords: Siganus sutor; reproduction; artisanal fisheries; Western Indian Ocean; fisheries management. 
$\mathrm{DOI}$

ARTIGO REVISTA BRASILEIRA DE ENGENHARIA DE PESCA

\section{Introdução}

O peixe coelho sapateiro, Siganus sutor (Valenciennes, 1835), é uma espécie da família Siganidae, comummente conhecida como peixe coelho e safi nas línguas locais (Figura 1). Com cerca de 13 espécies reconhecidas no Oceano Índico Ocidental (OIO), esta família é amplamente distribuída na região (Lam, 1974; Smith \& Heemstra, 1986; Woodland, 1990; Randall, 1995). Apesar de apresentar um tamanho relativamente pequeno, o S. sutor é muito procurado para a alimentação na região (Agembe, 2012).

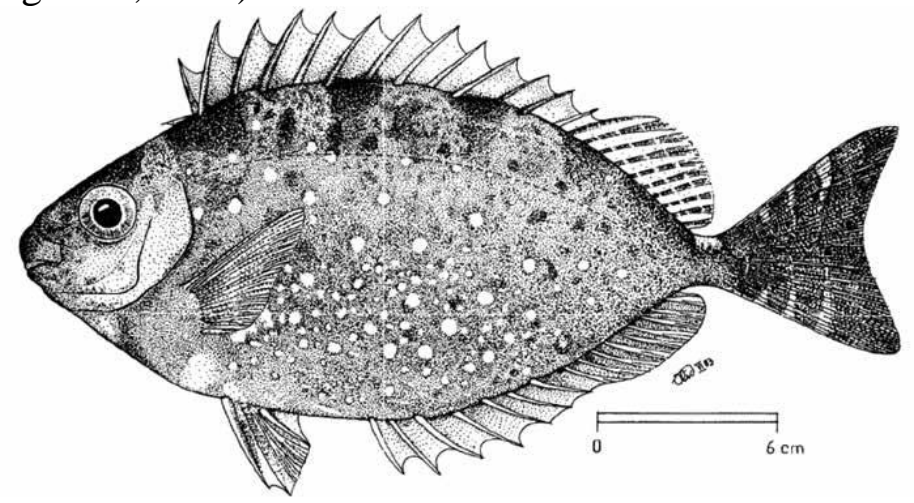

Figura 1. Ilustração do Siganus sutor (Woodland, 1984).

Ocorrendo em águas costeiras, associado a macrófitas e rochas e/ou recifes de coral a uma profundidade entre 1 a 12 m (Smith \& Heemstra, 1986; Fischer et al., 1990; Allen \& Erdmann, 2012), o Siganus sutor é uma espécie herbívora de pouca longevidade (2,5 anos) e de crescimento rápido (Grandcourt, 2002; Bijoux et al., 2013), sendo considerada uma das mais importantes espécies de peixes comerciais na pesca artesanal no OIO (Geets, Coene, \& Ollevier, 1997; Grandcourt \& Cesar, 2003; Bijoux et al., 2013). No Quénia e Seychelles, esta espécie é capturada por gaiola e linha de mão, sendo a mais abundante nas capturas artesanais (Robinson, Marguerite, \& Isidore, 2007; Wambiji et al., 2008; Robinson et al., 2011; Samoilys, Kanyange, Macharia, \& Maina, 2012; Maina, Samoilys, Alidina, \& Osuka, 2013; Bijoux et al. 2013; SCRFA, 2018).

Por sua vez, na Tanzânia, o $S$. sutor é capturado por uma variedade de artes de pesca como as redes de arrasto para praia, gaiolas e linha de mão (Woodland, 1984), onde cerca de 1,2 toneladas por ano são capturadas em Zanzibar (IUCN, 2019).

Em Moçambique, a captura do S. sutor é feita com redes de cerco, de emalhe, de arrasto para praia, gaiolas, linha de mão e armas submarina (Fischer et al., 1990; MGDP, 2016). Na província de Cabo Delgado esta espécie é uma das mais abundantes nas capturas artesanais (Bilika, Farooq, Simão, Soares, \& Morgado, 2019).

Estudos feitos no OIO apontam que esta espécie possui diferentes padrões de reprodução, apresentando uma desova contínua ou descontínua ao longo do ano em função das regiões geográficas. Por exemplo, Ntiba \& Jaccarini (1990) afirmam que o período de desova desta espécie ocorre de janeiro a fevereiro e de maio a junho, coincidindo assim com um à dois meses após o início de cada período das monções, entre setembro e junho nas Ilhas Seychelles (Bijoux et al., 2013), no Quénia ocorre entre janeiro a fevereiro, junho a julho e novembro a dezembro (Kamukuru, 2009; Maina et al., 2013). No OIO, esta espécie é conhecida por formar agregações reprodutivas (Ntiba \& Jaccarini, 1990; Bijoux et al., 2013; Maina et al., 2013). 
O tamanho da primeira maturidade para fêmeas e machos é de $228 \mathrm{~mm}$ e $217 \mathrm{~mm}$ (Comprimento total-Ct), respetivamente, e a fecundidade média para esta espécie é de $627,052 \pm 78,886$ oócitos por fêmea (Kamukuru, 2009). O comprimento máximo registado é de 450 mm $(\mathrm{Ct})$ para os dois sexos (Smith \& Heemstra, 1986; Fischer et al., 1990; Allen \& Erdmann, 2012).

Em Moçambique, estudos anteriores (Chaúca et al., 2013; Bilika et al., 2019) sobre $S$. sutor estimaram a abundância e a distribuição de frequência de tamanho de comprimento dos indivíduos, não há informação sobre a biologia reprodutiva da espécie, tais como: tamanho da primeira maturação, índice gonadossomático, fator de condição gonadal e estágios de maturação das gonadas. O conhecimento dos parâmetros reprodutivos, como o período de desova, poderá suportar esforços de maneio destinados a proteger os estágios críticos do ciclo de vida de Siganus sutor, incluindo as agregações reprodutivas.

O objetivo do presente trabalho foi identificar o período de reprodução através de análise de distribuição das classes de comprimento corporal e da primeira maturação, do índice gonadossomático, do fator de condição gonadal e do estágio de maturação. Espera-se, desta forma, compreender a dinâmica temporal da reprodução do Siganus sutor e os potenciais impactos da pesca artesanal sobre as populações desta espécie de modo a contribuir no delineamento de medidas de gestão e conservação desta espécie de importância comercial na região.

\section{Material e métodos}

\section{Área de estudo}

O distrito de Mecúfi está situado a sudeste da província de Cabo Delgado (Figura 2) e tem uma zona costeira com uma área de $70 \mathrm{~km}^{2}$ (Arquitectos sin Fronteras, Fundación Global Nature, SPDI de Mecúfi \& DPCAA de Cabo Delgado, 2012).

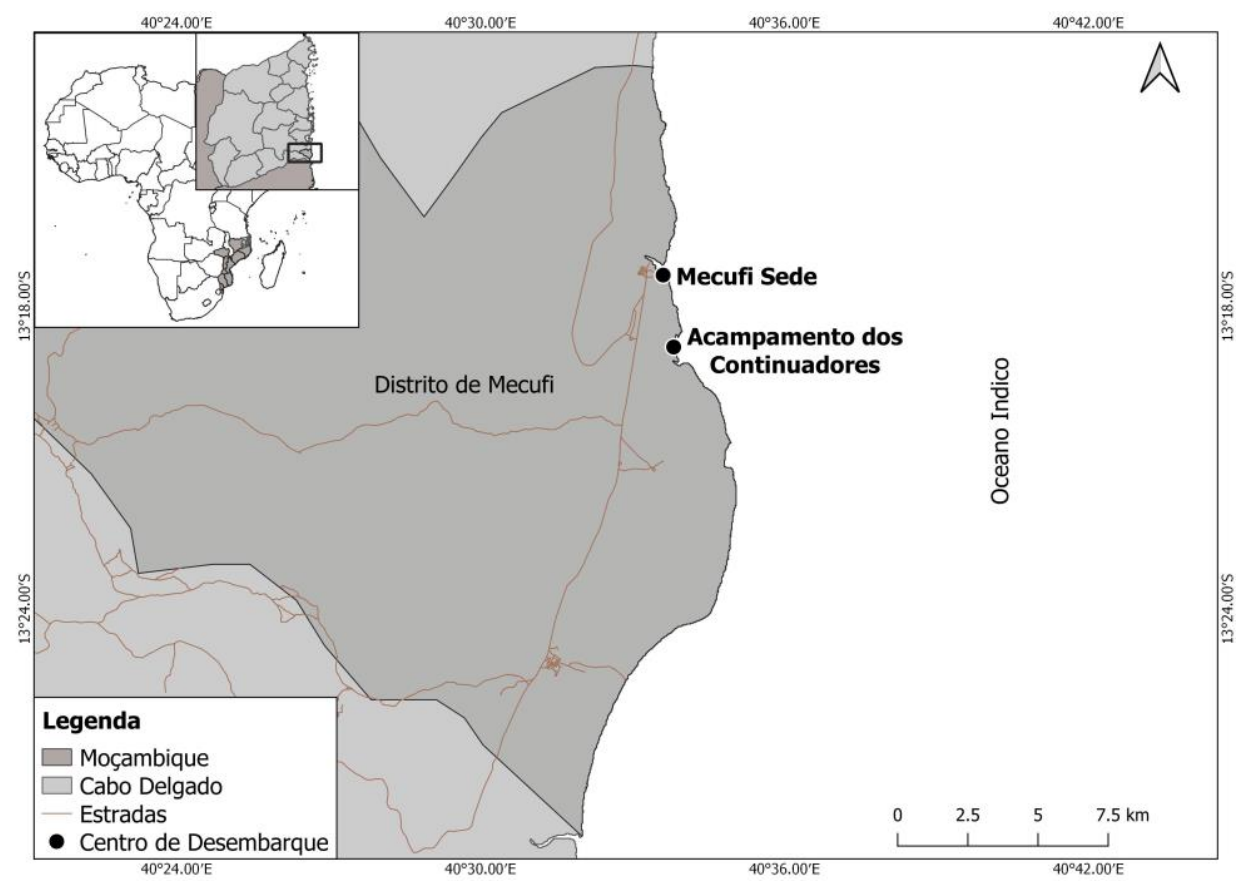

Figura 2. Mapa da localização geográfica da aldeia de Mecúfi e de outras referenciadas no estudo. 


\section{Coleta de dados}

Para obtenção dos dados biométricos e reprodutivos, os indivíduos foram coletados mensalmente nos centros de desembarques de Mecúfi-Sede e Acampamento dos Continuadores no distrito de Mecúfi, entre agosto de 2018 e julho de 2019, capturados pelos pescadores artesanais durante a maré de sizígia, maré com maior amplitude que ocorre durante as fases de lua nova e lua cheia, pois, segundo pescadores locais é na maré sizígia que se registam maiores capturas. De acordo com Hoguane et al. (2007), os pescadores artesanais detém um conhecimento empírico sobre a disponibilidade dos vários tipos de peixe e as artes de pesca a empregar em função das marés. Regra geral, ainda conforme estes autores, durante as marés sizígias, associadas às inundações e às correntes fortes, os pescadores pescam mais a montante dos estuários e nos pântanos de manguezais, e durante as marés quadratura, associadas ao baixo nível de água, os pescadores pescam nas baías e/ou no mar adjacente. Assim, durante a maré sizígia é esperado que o esforço de pesca no mar adjacente seja menor em relação a maré quadratura, e como resultado terse-á maior rendimento na maré sizígia (Cavariato \& Mualeque, 2013).

Os critérios para a escolha dos dois locais de desembarque foram baseados em sua história, sendo locais onde se desembarque mais capturas no distrito. Para composição amostral, os peixes foram selecionados aleatoriamente, de acordo com os métodos descritos por Baloi, Afonso, Premigi, e Volstad (2007) que consiste numa amostragem aleatória simples e representativa da composição específica total de cada embarcação, onde presume-se que todas unidades de pesca (embarcação, artes de pesca e espécies) desembarcadas num determinado centro têm a mesma probabilidade de pertencerem a amostra, isto é, de serem inquiridas ou amostradas.

Para cada exemplar foram tomados os dados do comprimento total $(\mathrm{Ct})$, em milímetros $(\mathrm{mm})$ no ictiómetro com precisão de $1 \mathrm{~mm}$ e o peso total $(\mathrm{Pt})$, em gramas $(\mathrm{g})$ na balança com $5 \mathrm{~g}$ de precisão (IDEAMART modelo B1-C05, máximo $50 \mathrm{~kg}$ e mínimo $20 \mathrm{~g}$ ). Por meio de uma incisão ventro-longitudinal, todos os exemplares foram abertos, removidas e pesadas as gonadas usando balança analítica de 0,01 g de precisão (MART modelo QC 08, máximo $200 \mathrm{~g}$ e mínimo 0,01 g).

Para além da identificação macroscópica do sexo, os estágios de maturação foram identificados usando uma escala de desenvolvimento variando de imaturos aos desovados/aspermo (imaturo, imaturo em desenvolvimento, maturação inicial, em maturação, maturação final, totalmente maturo e desovado/aspermo) de cordo com descrição de Ntiba \& Jaccarin (1990). Basicamente, critérios morfológicos externos (forma, cor, tamanho relativo, tamanho de oócitos e de volume dos ovários e testículos) foram usados para determinar o sexo e os estágios de maturidade.

\section{Análise de dados}

Para análise da estrutura dos tamanhos, os indivíduos capturados foram distribuídos em 10 classes de comprimento total de $30 \mathrm{~mm}$ de intervalos. A proporção entre os sexos foi analisada mensalmente e por classe de tamanho. Foi utilizado o teste Qui-quadrado $\left(\chi^{2}\right)$, com significância de $5 \%$, para comparar a proporção sexual em cada período amostrado para as diferentes classes de comprimento (Vazzoler, 1996). Verificada a não normalidade de dados com teste Shapiro-Wilk, foi usado posteriormente o método não paramétrico de teste Kruskal-Wallis, à 5\% de significância com o SPSS 17.0. 
$\mathrm{DOI}$

\section{ARTIGO REVISTA BRASILEIRA DE ENGENHARIA DE PESCA}

Com os dados referentes ao sexo, estágio de maturação e comprimento de cada indivíduo foi obtido o percentual de indivíduos maturos (considerando os estágios em maturação inicial, em maturação, maturação final, totalmente maturo e desovado/aspermo) por classe de comprimento para cada sexo, seguindo o proposto por Vazzoler (1996).

O tamanho médio da primeira maturação, $\mathrm{L}_{\mathrm{M} 50}$, o tamanho em que $50 \%$ das fêmeas ou machos estão reprodutivamente ativos durante os principais períodos de desova, foi ajustada uma curva logística, usando o método dos mínimos quadrados, à proporção de indivíduos macroscopicamente maturos considerado como variável dependente $(Y)$ por classes de tamanho de comprimento como variável independente (X) (Kolding \& Skålevik, 2010).

Esse método, denominado como maturidade efetiva, reconhece que, apesar da maturidade, nem todos indivíduos são reprodutivamente ativos na época da desova (Pears, Choat, Mapstone, \& Begg, 2006), minimizando erros na designação de peixes imaturos como indivíduos inativos (Samoilys \& Roelofs, 2000).

A evolução da proporção de indivíduos adultos, denominada ogiva de maturação, com o comprimento foi estimado em classes de comprimento de $10 \mathrm{~mm}$ de intervalos, ajustada por um modelo de regressão não linear do total de indivíduos em cada classe, descrito por Duponchelle \& Panfili (1998):

$$
\% F M=\frac{1}{1+e^{-a\left(L-L_{M 50}\right)}}
$$

Onde \%FM é a percentagem do peixe maturo (fêmea ou macho) por classe de comprimento, L é o comprimento médio de cada classe, e $a$ e L ${ }_{\text {M50 }}$ são constantes de modelo.

O índice gonadossomático (IGS), que representa a contribuição da massa das gonadas em relação a massa corporal total, foi determinado em ambos os sexos por meio da equação (Agembe, 2012):

$$
I G S=\frac{P g}{P t-P g} * 100
$$

Onde IGS representa o índice gonadossomático em percentual; Pg o peso da gónada (g) e Pt o peso total do peixe $(\mathrm{g})$.

Foi determinada a frequência relativa percentual $(\%)$ para cada estágio de maturação, de indivíduos maturos ao desovado/aspermo (IIb à VI), e produzidos histogramas em todos os meses de coleta, com o objetivo de identificar a época de desova do Siganus sutor (Vazzoler, 1996). De acordo com Ntiba \& Jaccarin (1990), são considerados peixes maturos a partir de maturação inicial (estágio IIb). As gonadas que não foram possíveis as identificações de estágios de maturação foram excluídas das análises. A percentagem relativa percentual mensal de estágios de maturação foi relacionada com os dados de precipitação do distrito de Mecúfi adquiridos no Instituto Nacional de Meteorologia. Segundo Fonteles-Filho (2011), a precipitação é considerado um dos fatores que funciona como estímulo para o início da desova.

\section{Resultados}

No período de agosto de 2018 a julho de 2019 foram amostrados 724 indivíduos, dos quais 366 fêmeas, 239 machos e 119 juvenis não foi possível a identificação do sexo (Figura 3). 
$\mathrm{DOI}$

O comprimento total médio do $S$. sutor também variou significativamente entre os meses, segundo o teste do Kruskal-Wallis $\left(\chi_{\text {calc }}^{2}=149,69 ; d f=10 ; p<0,05\right)$ com a menor média de comprimento registada em setembro e maior no mês de abril e julho (Figura 4).

$\mathrm{O}$ teste do Qui-quadrado mostrou que a frequência de fêmeas e machos por classe de comprimento apresentou diferenças significativas apenas em quatro das 10 classes definidas, com predominância das fêmeas no menor tamanho entre classes de 80 e $290 \mathrm{~mm} \mathrm{de} \mathrm{Ct}$, enquanto os machos predominaram classes de maior tamanho entre 290 e $350 \mathrm{~mm}$ de $\mathrm{Ct}$.

No geral, a dominância dos indivíduos amostrados foi evidente para as fêmeas (1,5 fêmeas: 1 macho; $\chi^{2}$ calc $\left.=31,30 ; p<0,05\right)$, com enfoque os meses de janeiro, fevereiro, maio, julho, outubro, novembro e dezembro $\left(\chi^{2}\right.$ calc $\left.=21,71 ; p<0,05\right)$ (Figura 5$)$.

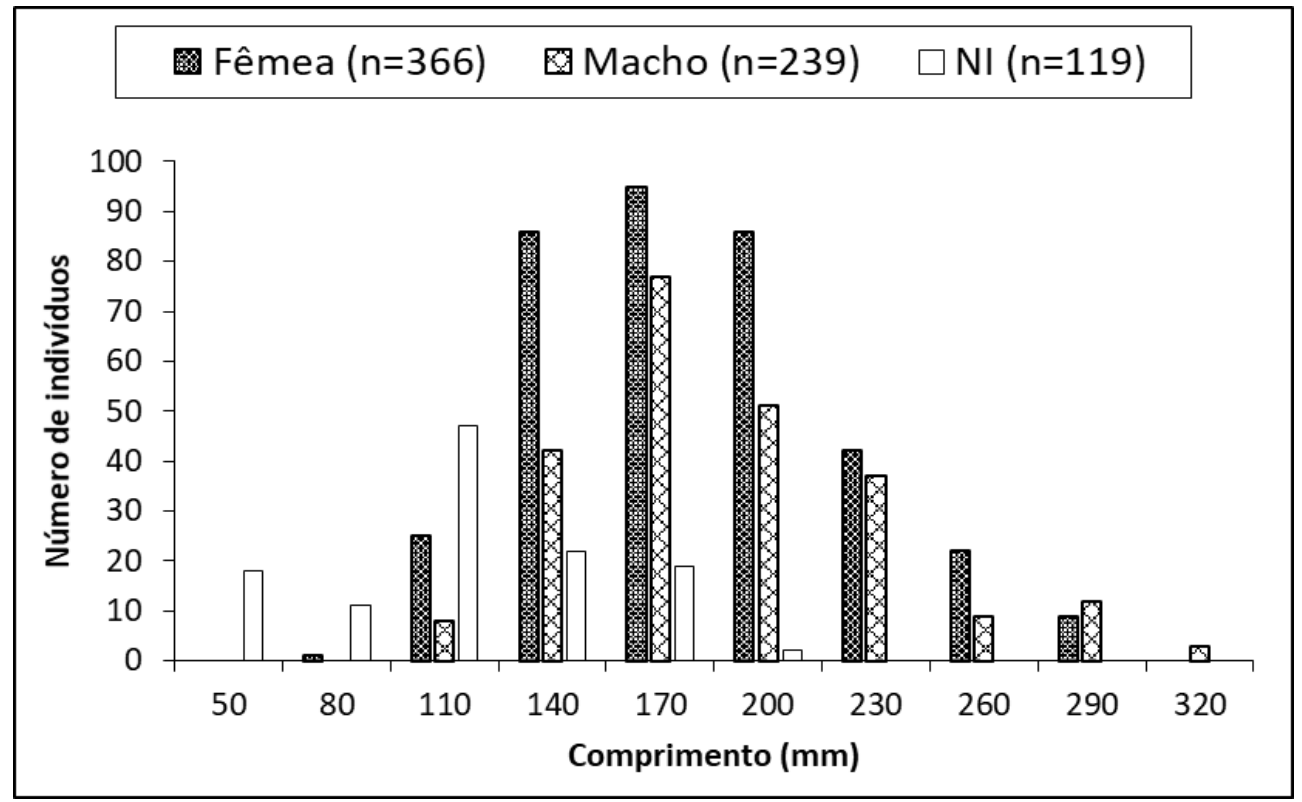

Figura 3. Distribuição de frequência por classes de comprimento total de fêmeas e machos do peixe coelho sapateiro, Siganus sutor, para o período de agosto de 2018 a julho de 2019 (dados não coletados em março). $\mathrm{NI}=$ indivíduos com o sexo não identificado. 
$\mathrm{DOI}$

\section{ARTIGO REVISTA BRASILEIRA DE ENGENHARIA DE PESCA}

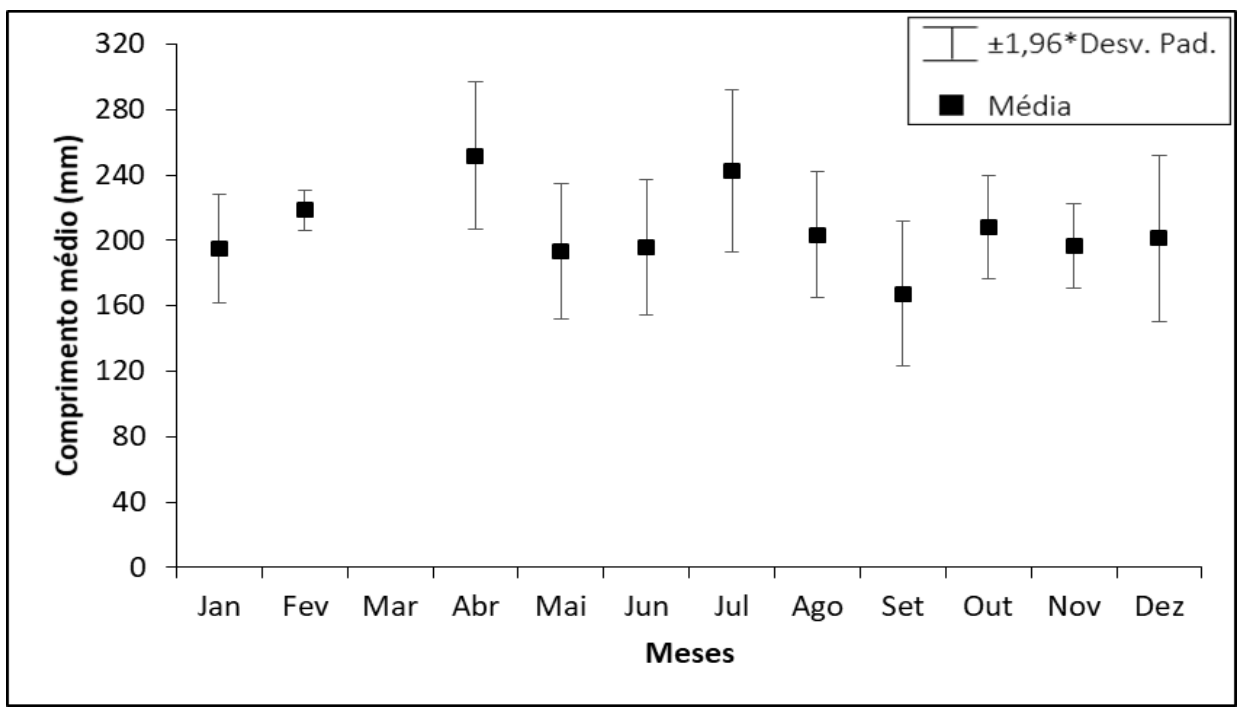

Figura 4. Variações mensais do comprimento total médio do Siganus sutor capturado no distrito de Mecúfi para o período de agosto de 2018 a julho de 2019 (dados não coletados em março). As barras indicam o desvio padrão (SD) da média.

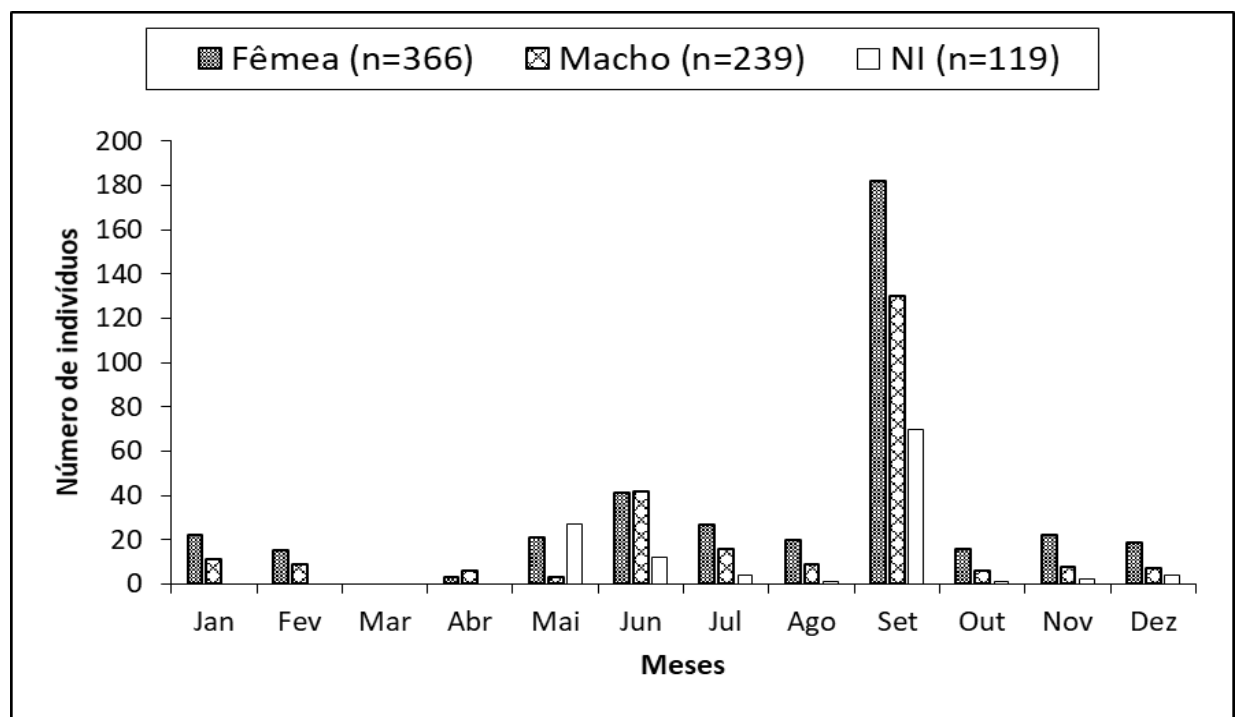

Figura 5. Distribuição de frequência mensal de fêmeas e machos do Siganus sutor, entre Agosto de 2018 a Julho de 2019 (dados não coletados em março). NI=indivíduos com o sexo não identificado.

O comprimento médio de primeira maturação sexual ( $\left.\mathrm{L}_{\mathrm{M} 50}\right)$ foi de 187,01 mm para fêmeas $\left(\mathrm{Y}=0,4459 \mathrm{X}-17,923, \mathrm{R}^{2}=0,9169\right)$ e $201,73 \mathrm{~mm}$ para machos $\left(\mathrm{Y}=0,4526 \mathrm{X}-21,395, \mathrm{R}^{2}=0,9216\right)$. A partir de 240 e $270 \mathrm{~mm}$ de comprimento total para fêmeas e machos respetivamente, todos os indivíduos são considerados maturos, participantes activos do processo reprodutivo (Figura 6).

O maior valor do índice gonadossomático (IGS) foi observado no mês de abril, com tendências de aumento entre junho a setembro (Figura 7). Por outro lado, os menores valores foram verificados entre janeiro a fevereiro, e no mês de maio. 


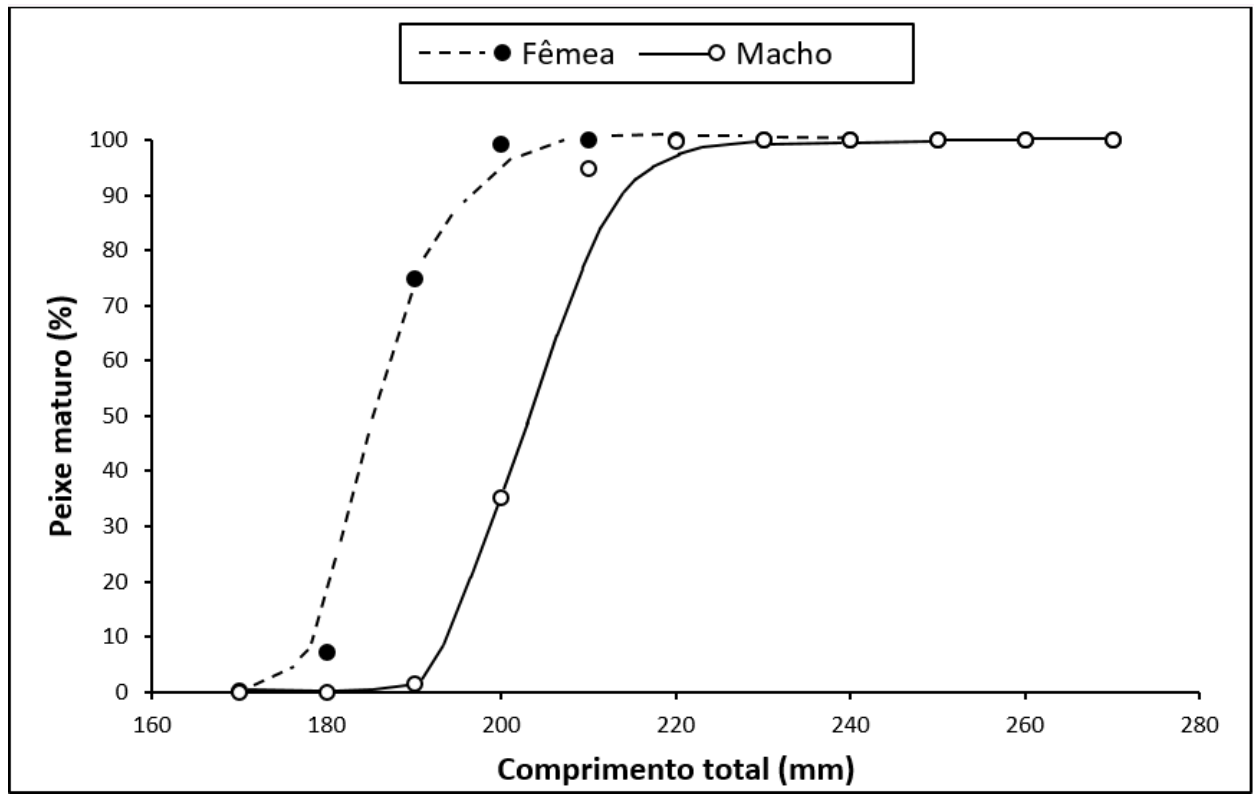

Figura 6. Percentagem observada de fêmeas e machos maturos do Siganus sutor por classes de tamanho de $10 \mathrm{~mm}$ de intervalos de $\mathrm{Ct}$ ajustadas a uma função logística estimada a partir do tamanho da primeira maturação sexual, dado como 187,01 e 201,73 mm Ct, respetivamente.

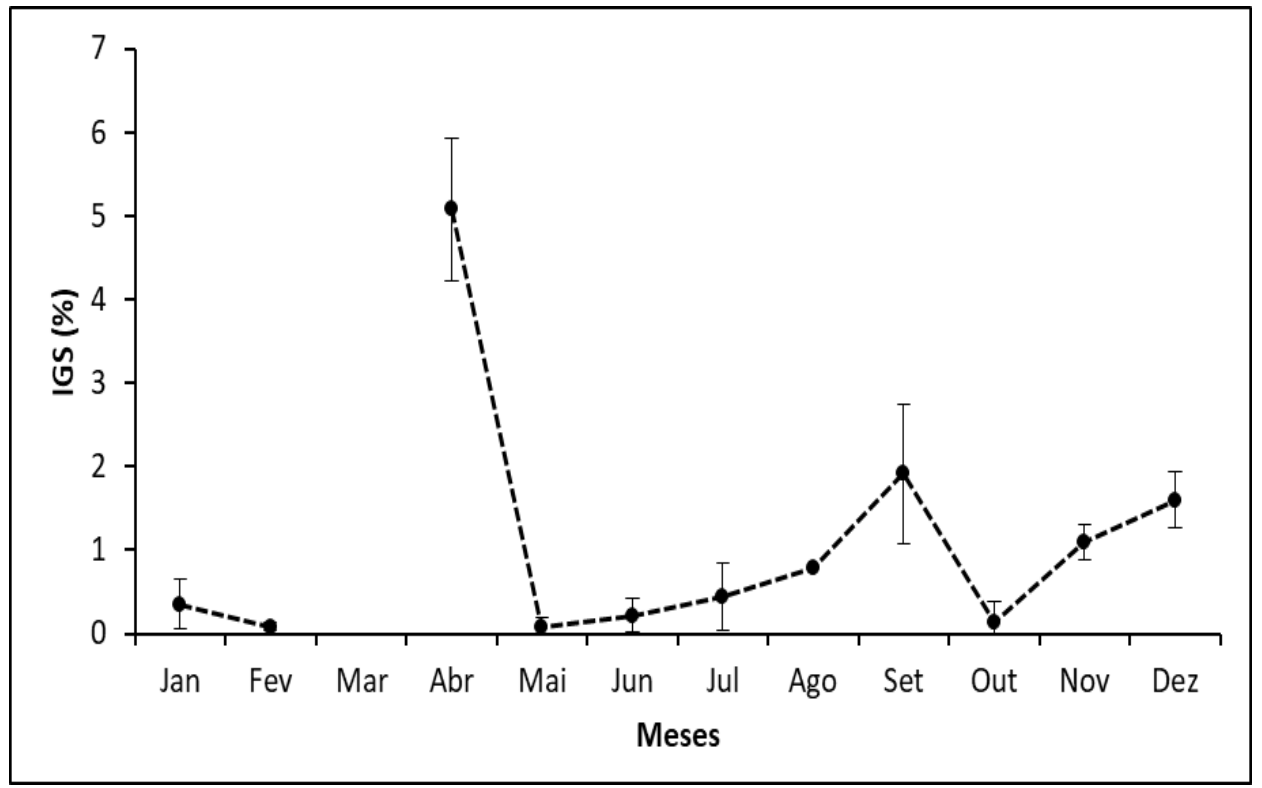

Figura 7. Variação média mensal do índice gonadossomático do Sigaus sutor no distrito de Mecúfi, entre agosto de 2018 e julho de 2019 (dados não foram coletados em março). As barras indicam o desvio padrão da média.

A partir da análise macroscópica das gonadas, foram identificados sete estágios de maturação gonadal: imaturo, imaturo em desenvolvimento, maturação inicial, em maturação, maturação final, totalmente maturo e desovado/aspermo. O maior número de indivíduos maturos foi verificado nos meses de abril e de junho-setembro, coincidindo com os menores picos de precipitação. $O$ período de repouso, com presença de menor quantidade de indivíduos com maturação final, totalmente 
$\mathrm{DOI}$

maturo e desovado/aspermo foi verificado entre outubro-fevereiro incluindo mês de maio, período de maior precipitação (Figura 8).

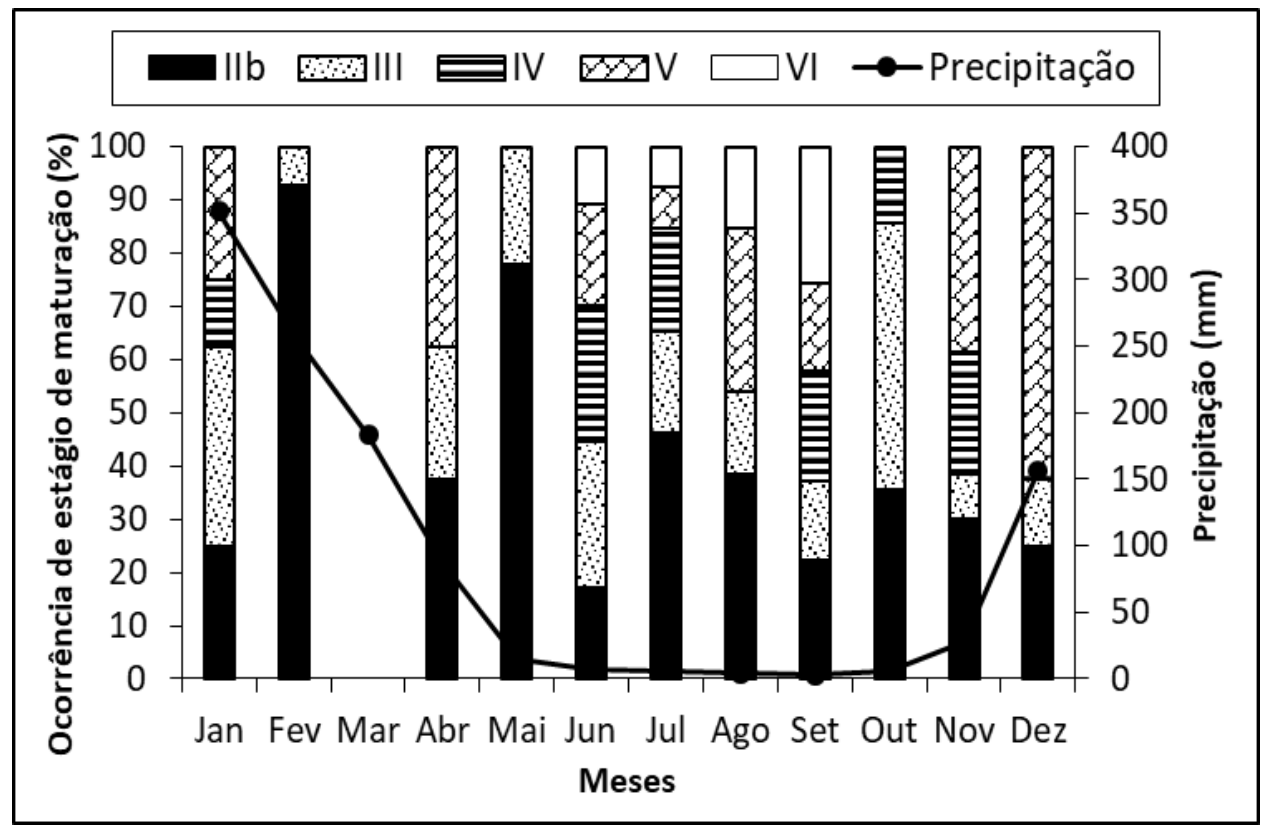

Figura 8. Variação mensal na proporção dos estágios de maturação dos indivíduos maturos do Siganus sutor e precipitação no distrito de Mecúfi, entre agosto de 2018 e julho de 2019 (IIb=maturação inicial, III=em maturação, IV=maturação final, $V=$ =totalmente maturo e $V I=$ desovado/aspermo). Os dados não foram coletados em março. Os indivíduos imaturos (estágios I=imaturo e IIa=imaturo em desenvolvimento) foram excluídos da representação gráfica.

\section{Discussão}

O padrão de distribuição por classes de comprimentos do Siganus sutor observado na presente pesquisa é semelhante aos comprimentos verificados para mesma espécie no Quénia por Wimbiji (2013). Os resultados mostraram a predominância de maiores tamanhos para os machos, em detrimento das fêmeas, o que provavelmente pode estar relacionado com o maior gasto energético realizado pelas fêmeas durante o processo reprodutivo, enquanto os machos investem a energia no crescimento, ou seja, aumento da biomassa corporal (peso e/ou tamanho) (Huntingford, Chellappa, Taylor, \& Strang, 2001).

O estudo demonstrou a variação do comprimento médio entre os meses, mostrando a captura de indivíduos relativamente maiores nos meses de fevereiro, abril, julho, agosto, outubro e dezembro, com comprimentos médios acima de $200 \mathrm{~mm}$, e os restantes meses com comprimentos médios menores que $200 \mathrm{~mm}$. Estudos realizados em várias partes do mundo, por diferentes autores mostraram que os tamanhos capturados são semelhantes ou inferiores: Laroche \& Ramananarivo (1995), na região de Tulear, sudoeste de Madagáscar registou menores tamanhos (<100 mm) de peixes coelhos (Siganidae), Kamururu (2009), na Reserva Marinha de Dar-es-Salaam (RMDs), Tanzânia registou comprimentos médios mensais acima de $200 \mathrm{~mm}$, dados esses encontrados também no Quénia nas capturas de gaiola (Wimbiji, 2013). Tamanhos reduzidos dos indivíduos em Mecúfi podem estar associados ao efeito de sobre-exploração deste recurso na região (CarvalhoNeta \& Castro, 2008) embora seja parte da região ainda considerada de capturas de peixe comparativamente altas para a África Oriental (Samoilys et al., 2019). 
A proporção sexual do $S$. sutor apresentou predominância de fêmeas praticamente todo o ano, com excepção de abril. De acordo com Nascimento, Yamamoto, \& Chellappa (2012) vários fatores podem atuar na determinação da proporção sexual nos peixes. Ainda conforme estes autores, a mortalidade, o crescimento e o comportamento são exemplos de fatores que, atuam de forma diferenciada sobre os sexos, que pode alterar a proporção sexual em diversas fases de desenvolvimento.

A maioria das espécies tende a apresentar uma proporção sexual de 1:1, mas esta pode variar consideravelmente entre as espécies, populações de uma mesma espécie em diferentes meses, classes de comprimento e/ou anos dentro de uma mesma população, com predomínio, por exemplo, de machos ou fêmeas (Nascimento et al., 2012; Santos, Silva, Costa, \& Araújo, 2015). Por outro lado, isso pode ser atribuído a uma série de fatores incluindo a vulnerabilidade das fêmeas às artes de pesca e apresentando movimentos migratórios diferentes em comparação com os machos que permitem que sejam mais facilmente capturadas (Abderrazik, Baali, Schahrakane, \& Tazi, 2016; Sululu, Kamukuru, Sekadende, Mahongo, \& Igulu, 2020), e mortalidade natural diferente entre os sexos (Turner, Grimes, \& Able, 1983). Alguns estudos indicam que as diferenças na proporção sexual são difíceis de descrever, mas alguns autores atribuem isso a uma combinação de fatores, incluindo a disponibilidade de alimentos na região, como citados em vários estudos (Nascimento et al., 2012; Santos et al., 2015; Sululu et al., 2020), que relataram que quando o alimento é abundante em uma determinada área, as fêmeas predominam, e a situação é diferente quando o alimento é limitado. A variação mensal observada na proporção sexual do Siganus sutor no presente estudo pode ser atribuída a um ou a uma combinação de todos esses fatores.

O tamanho médio de primeira maturação em ambos os sexos encontrado na região para esta espécie foi inferior ao registado na Tanzânia e no Quénia (ver Tabela 1). Essa diferença pode ser um indício de que os indivíduos da costa de Mecúfi atingiram a maturação precocemente na tentativa de recuperar o estoque face à exploração excessiva (sobre-exploração de crescimento) desta espécie na região (Carneiro \& Castro, 2005).

O comprimento médio da primeira maturação gonadal é importante para o uso racional dos estoques, pois, é um aspeto crucial para fixação dos tamanhos mínimos de captura e para a determinação da seletividade de artes, isto é, de tamanho de malhas e de anzóis (Araújo \& Chellappa, 2002). Sendo este mais um sinal de alerta para a possível sobre-exploração desta espécie no distrito.

O índice gonadossomático reflete o grau de maturidade dos peixes (Isaac-Nahum \& Vazzoler, 1987; Araújo \& Chellappa, 2002). Diante disto, a análise deste parâmetro associado aos estágios de maturação das gonadas permitiu identificar picos de desova do $S$. sutor no período de menor intensidade de precipitação, de acordo com Fonteles-Filho (2011) que considera esta uma das variáveis climática mais importante, pois funciona como estímulo para o início da desova. De acordo com Gurgel, Canan, Nascimento, \& Chellappa (2013), estudando Stegastes fuscus, durante a seca as caraterísticas ambientais tornam mais favoráveis para maturação das gonadas, corte, construção de ninhos, desova e sobrevivência.

Sendo assim, a ocorrência de peixes maturos, totalmente maturos e desovados/aspermo entre junho e setembro e o maior pico do índice gonadossomático em abril, acompanhada de uma diminuição gradual da ocorrência de precipitação, foram consistentes com período de desova do $S$. sutor. O ligeiro declínio nos valores de IGS (maio-agosto) observados durante o pico de desova desta espécie indica alto gasto de energia durante a desova (Rodrigues-Filho, Verani, Peret, Sabinson, \& Branco, 2011) o que provavelmente resultou em más condições de saúde dos peixes (Bhattacharya \& Sree, 2012). Isso pode resultar em efeitos menores sobre os ovos recém- 
fertilizados, taxas de eclosão e sobrevivência larval desta espécie (Souza-Conceição, RodriguesRibeiro, \& Castro-Silva, 2005; Sululu et al., 2020).

Estudos anteriores indicam que variações no período de desova da espécie são resultantes de um conjunto de fatores ambientais encontrados em cada local, as quais podem ser ideais para a desova por um período significativo do ano de acordo com a região estudada (Santos et al., 2015). Contudo, de acordo com Fonteles-Filho (2011), fatores como precipitação, temperatura, salinidade, fotoperíodo ou abundância de alimentos funcionam como estímulo para o início da desova, fatores estes sugeridos pelos Takemura, Rahman, Nakamura, Park, \& Takano (2004), na sazonalidade reprodutiva de peixes coelho. O mesmo período de desova foi reportado pelos pescadores no local em estudo (Machaieie \& Silva, 2020). No entanto, foi reportado para a região uma variação enorme na sazonalidade, tamanho mínimo e médio da primeira maturidade do Siganus sutor, como é possível observar na Tabela 1. Por um lado, as diferenças verificadas na sazonalidade podem estar relacionadas às diferenças de padrões de ventos e precipitação na região (URT \& WWF, 2005; Olendo et al., 2019), por ambos serem considerados alguns dos fatores ambientais que podem influenciar na reprodução (Johannes, 1978; Heyman, Kjerfve, Graham, Rhodes, \& Garbutt, 2005; Choat, 2012; Kobara, Heyman, Pittman, \& Nemeth, 2013; Rosli, Ibrahim, \& Masron, 2014). Por outro lado, as diferenças relativamente aos tamanhos da primeira maturidade podem estar relacionadas com a utilização de diferentes métodos para estimar o tamanho de maturação.

Tabela 1. Sazonalidade, tamanho mínimo e médio $\left(\mathrm{L}_{\mathrm{M} 50}\right)$ da primeira maturidade sexual do Siganus sutor de estudos anteriores e do presente estudo.

\begin{tabular}{|c|c|c|c|}
\hline Referência & Local & IGS - Período de Picos & $\begin{array}{l}\text { Comprimento de } \\
\text { maturidade }\end{array}$ \\
\hline De Souza (1988) & Mombasa (Quénia) & outubro-janeiro & $\begin{array}{l}240 \text { mm Ct-fêmeas } \\
240 \text { mm Ct-machos }\end{array}$ \\
\hline Ntiba \& Jaccarini (1988) & Mombasa (Quénia) & janeiro/fevereiro e maio/junho & $217 \mathrm{~mm} \mathrm{Ct}-$ machos \\
\hline Kamukuru (2006) & $\begin{array}{l}\text { Dar-es-Salaam } \\
\text { (Tanzânia) }\end{array}$ & dezembro-maio & 228 mm Ct-fêmeas \\
\hline Kamukuru (2009) & $\begin{array}{l}\text { Dar-es-Salaam } \\
\text { (Tanzânia) }\end{array}$ & outubro-maio & $\begin{array}{l}227,7 \text { mm Ct-fêmeas 217,1 } \\
\text { mm Ct-machos }\left(\mathrm{L}_{\mathrm{M} 50}\right)\end{array}$ \\
\hline Agembe (2012) & $\begin{array}{l}\text { Msambweni } \\
\text { (Quénia) }\end{array}$ & $\begin{array}{l}\text { novembro e janeiro }(>80 \%) \\
\text { junho, julho e outubro }(60-70 \%)\end{array}$ & 282 mm Ct-fêmeas ( $\left.\mathrm{L}_{\mathrm{M} 50}\right)$ \\
\hline Este estudo & $\begin{array}{l}\text { Mecúfi } \\
\text { (Moçambique) }\end{array}$ & abril-setembro & $\begin{array}{l}187,01 \mathrm{~mm} \mathrm{Ct} \text {-fêmeas } \\
201,73 \mathrm{~mm} \mathrm{Ct} \text {-machos }\left(\mathrm{L}_{\mathrm{M} 50}\right)\end{array}$ \\
\hline
\end{tabular}

\section{Conclusão}

O presente estudo permitiu conhecer aspetos importantes de reprodução da população do Siganus sutor no distrito de Mecúfi. A proporção sexual é favorável às fêmeas, sendo que os machos atingem tamanhos maiores do que as fêmeas. A variação do IGS nesta região, pode ser utilizado como indicativo para a(s) época(s) de desova entre abril e agosto. Cinquenta por cento da população do $S$. sutor, encontra-se adulta com cerca de 187,01 e 201,73 mm de comprimento total para fêmeas e machos respetivamente $\left(\mathrm{L}_{\mathrm{M} 50}\right)$, sendo assim, é necessário monitorar os tamanhos das malhas e anzóis das artes de pesca utilizadas de forma a evitar a captura de indivíduos menores que $202 \mathrm{~mm}$ para fêmeas e machos. Sugere-se ainda, novos estudos sobre a reprodução ao longo da costa de Cabo Delgado e de outras espécies de valor comercial na região, para garantir o correto ordenamento pesqueiro e contribuírem para uma legislação mais eficiente na conservação dos recursos pesqueiros. 


\section{Agradecimentos}

Aos membros dos conselhos comunitários de pescas e os pescadores por auxiliarem e colaborarem na obtenção das amostras nos centros de desembarques.

\section{Referências}

Abderrazik, W., Baali, A., Schahrakane, Y., \& Tazi, O. (2016). Study of reproduction of sardine, Sardina pilchardus in the North of Atlantic Moroccan area. AACL Bioflux, 9: 507-517.

Agembe, S. (2012). Estimation of important reproductive parameters for management of the Shoemaker spinefoot rabbitfish (Siganus sutor) in Southern Kenya. International Journal of Marine Science, 2(4): 24-30. [doi:10.5376/ijms.2012.02.0004]

Allen, G. R., \& Erdmann, M. V. (2012). Reef fishes of the East Indies. Tropical Reef Research, Perth, Australia.

Araújo, A. S., \& Chellappa, S. (2002). Estratégia reprodutiva do peixe voador, Hirundichthys affinis Günther (Osteichthyes, Exocoetidae). Revista Brasileira de Zoologia, 19(3): 691-703.

Arquitectos sin Fronteras, Fundación Global Nature, SPDI de Mecúfi, \& DPCAA de Cabo Delgado. (2012). Diagnóstico sobre o estado de conservação do ecossistema de mangal da vida Sede de Mecúfi. Proposta de intervenção para sua exploração sustentável. AECID, Dip. Palencia. Mecúfi, Moçambique, $82 \mathrm{pp}$.

Baloi, A. P., Afonso, P. S., Premigi, N., \& Volstad, J. H. (2007). Metodologia de colheita e processamento de dados de captura e esforço da pesca artesanal em Moçambique. Revista de Investigação Pesqueira $n^{o}$ 25. Instituto Nacional de Investigação Pesqueira; MaputoMoçambique, $29 \mathrm{pp}$.

Bhattacharya, P., \& Banik S. (2012) Length-weight relationship and condition factor of the pabo catfish Ompok pabo (Hamilton, 1822) from Tripura, India. Indian Journal of Fisheries, 59: 141-146.

Bijoux, J. P., Dagorn, L., Berke, G., Cowley, P. D., Sorla, M., Gaertner, J., \& Robinson, J. (2013). Temporal dynamics, residency and site fidelity of spawning aggregations of a herbivorous tropical reef fish Siganus sutor. Marine Ecology Progress Series, 475(1): 233-247.

Bilika, F., Farooq, H., Simão, C., Soares, A., \& Morgado, F. (2019). Composição específica da comunidade de peixes da Baía de Pemba (Norte Moçambique). Seminário Internacional. Alterações Climáticas, Biodiversidade e Sociedade: Desafios no contexto Africano. CAPTAR, 8(2): 144-155.

Carneiro, M. H., \& Castro, P. M. G. Estoque Sudeste. In: Cergole, M. C., Ávila-Da-Silva, A. O. \& Rossi-Wongtschowski, C. B. (eds.) (2005). Análise das principais pescarias comerciais da região Sudeste-Sul do Brasil: dinâmica populacional das espécies em explotação. Instituto Oceanográfico (USP): São Paulo, 22-35 pp.

Carvalho-Neta, R. N. F., \& Castro, A. C. L. (2008). Diversidade das assembleias de peixes estuarinos da Ilha dos Caranguejos, Maranhão. Arquivos de Ciências do Mar, 41(1): 48-57.

Cavariato, E. C., \& Mualeque, D. O. (2013). Relação entre o ciclo da maré e rendimentos de Thryssa vitrirostris (Ocar de cristal), Sillago sihama (Pescadinha comum) e Sardinela albella capturados por arrasto a praia no distrito de Angoche, norte de Moçambique. Instituto Nacional de Investigação Pesqueira. Revista Moçambicana de Investigação Pesqueira, RIP 34: 2-13. 
Chaúca, I., Limited, P., Pereira, T., Chacate, O., Mualeque, D., Mutombene, R., Simango, A., Morais, E., Maúnde, C., Thuzine, A., Wetimane, A., Masquine, Z., Inácio, A., Leong, E., Samucidine, K., \& Alvaro, R. (2013). O estado de exploração dos recursos acessíveis à pesca artesanal em Moçambique - 2010. Instituto Nacional de Investigação Pesqueira. Boletim de Divulgação $n^{\circ} 54$.

Choat, J. (2012). Spawning Aggregations in Reef Fishes; Ecological and Evolutionary Processes. In book: Reef Fish Spawning Aggregations: Biology, Research and Management. doi:10.1007/978-94-007-1980-4_4

De Souza, T. F. (1988). Reproduction, length-weight relationship and condition factor in Siganus sutor (Valenciennes, 1835) (Pisces: Siganidae) from the Kenyan waters of the western Indian Ocean. Kenya Journal of Science Biology, 9: 89-101.

Duponchelle, F., \& Panfili, J. (1998). Variations in age and size at maturity of female Nile tilapia, Oreochromis niloticus, populations from manmade lakes of Côte d'Ivoire. Environmental Biology of Fish, 52: 453-465.

Fonteles-Filho, A. A. (2011). Oceanografia, biologia e dinâmica populacional de recursos pesqueiros. Expressão Gráfica Editora, Fortaleza, 464 pp.

Fischer, W., Silva, C., De Freitas, A., Poutiers, J. M., Schneider, W., Borges, T. C., Féral, J. P., \& Massinga, A. (1990). Guia de campo das espécies comerciais marinhas e de águas salobras de Moçambique. Fichas FAO de Identificação de Espécies para Actividades de Pesca, Roma, 369 pp.

Geets, A., Coene, H., \& Ollevier, F. (1997). Ecotparasites of the Whitespotted rabbitfish, Siganus sutor (Valenciennes, 1835) off the Kenyan coast: distribution within the host population and site selection on the gills. Parasitology, 115(1): 69-79.

Grandcourt, E. M. (2002). Demographic characteristics of a selection of exploited reef fish from the Seychelles: preliminary study.

Grandcourt, E. M., \& Cesar, H. S. J. (2003). The bio-economic impact of mass coral mortality on the coastal reef fisheries of the Seychelles. Fisheries Research, 60(1): 539-550.

Gurgel, L. L., Canan, B., Nascimento, W. S., \& Chellappa, S. (2013). Estrutura populacional e período reprodutivo de peixe donzela Stegastes fuscus (Cuvier, 1830) das poças de maré da praia de Búzios, Rio Grande do Norte, Brasil. Biota Amazônia, Macapá, 3(1): 85-93.

Heyman, W. D., Kjerfve, B., Graham, R. T., Rhodes, K. L., \& Garbutt, L. (2005). Spawning aggregations of Lutjanus cyanopterus (Cuvier) on the Belize Barrier Reef over a 6 year period. Jour. Fish Biol. 67: 83-101. doi:10.1111/j.1095-8649.2005.00714.x

Hoguane, A. M., Dove, V. F., Baquete, E., Nuvunga-Luis, R., Ibraimo, D., Rafael, R., Cuamba, B., \& Tsamba, A. J. (2007). Perfil diagnóstico da zona costeira de Moçambique. Revista de Gestão Costeira Integrada, 7(1):69-82.

Huntingford, F. A., Chellappa, S., Taylor, A. C., \& Strang, R. H. C. (2001). Energy reserves and reproductive investment in male three Spined sticklebacks, Gasterosteus aculeatus. Ecology of Freshwater Fish, 10(2): 111-117.

Isaac-Nahum, V. J., \& Vazzoler, A. E. A. de M. (1987). Biologia reprodutiva de Micropogonias furnieri (Desmarest, 1823) (Teleostei, Sciaenidae). 2. Relação gonadossomática, comprimento e peso dos ovários como indicadores do período de desova. Boletim do Instituto Oceanográfico, 35(2): 123-134.

IUCN (International Union for Conservation of Nature) (2019). The IUCN Red List of Threatened Species. Disponível em: www.iucnredlist.org. [Acessado em 11 de Julho de 2019] 
Johannes, R. E. (1978). Traditional marine conservation methods in oceania and their demise. Annual Review of Ecol. Sys. 9: 349-364.

Kamukuru, A. T. (2006). Reproductive biology of the Whitespotted rabbitfish, Siganus sutor (Pisces: Siganidae) from basket trap fishery in Dares Salaam marine reserve systems, Tanzania. Western Indian Ocean Marine Sciences Association, MARG-I/2006-01, Zanzibar, Tanzania, $31 \mathrm{pp}$.

Kamukuru, A. T. (2009). Trap fishery and reproductive biology of the Whitespotted rabbitfish Siganus sutor (Siganidae), within the Dar es Salaam Marine Reserves, Tanzania. Western Indian Ocean Journal of Marine Science, 8(1): 75-86.

Kobara, S., Heyman, W.D., Pittman, S.J., \& Nemeth, R.S. (2013). Biogeography of transient reeffish spawning aggregations in the Caribbean: a synthesis for future research and management. Oce. Mar. Bio. An Annual Review, 51: 281-326.

Kolding, J., \& Skålevik, A. (eds.) (2010). Pasgear II Version 2.4. University of Bregen. Bregen, Norway.

Lam, T. J. (1974). Siganids: Their biology and mariculture potential, Aquaculture, 3: 325-354. [http://dx.doi.org/10.1016/0044-8486(74)90001-5]

Laroche, J., \& Ramananarivo, N. (1995). A preliminary survey of the artisanal fishery on coral reefs of the Tulear Region (southwest Madagascar). Coral Reefs, 14: 193-200.

Machaieie, S., \& Silva, I. M. (2020). Spawning aggregations of fish in Cabo Delgado, Northern Mozambique: An interview-based survey of artisanal fishers. Western Indian Ocean Journal of Marine Science, 19(1): 33-43.

Maina, G. W., Samoilys, M. A., Alidina, H., \& Osuka, K. (2013). Targeted fishing of Siganus sutor spawning aggregations in southern Kenya. In book: Reef Fish Spawning Aggregations in the Western Indian Ocean: Research for Management. Edition: WIOMSA Book Series 13. Chapter: 3. Publisher: WIOMSA/SIDA/SFA/CORDIO.

MGDP (Mozambique Gas Development Project) (2016). Plano de Reassentamento. Esboço Final para Aprovação do Governo. Anexo B: Plano de Restabelecimento dos Meios de Subsistência Pesqueiros. Governo do Distrito de Palma.

Nascimento, W. S., Yamamoto, M. E., \& Chellappa, S. (2012). Proporção sexual e relação pesocomprimento do peixe anual Hypsolebias antenori (Cyprinodontiformes: Rivulidae) de Poças Temporárias da Região Semiárida do Brasil. Biota Amazônia, Macapá, 2(1), 37-44.

Ntiba, M. J., \& Jaccarini, V. (1988). Age and growth parameters of Siganus sutor in Kenyan marine inshore water, derived from numbers of otolith microbands and fish lengths. Journal of Fish Biology, 33: 465-470.

Ntiba, M. J., \& Jaccarini. V. (1990). Gonad maturation and spawning times of Siganus sutor off the Kenya coast: evidence for definite spawning seasons in a tropical fish. Journal of Fish Biology, 37(1): 315-325.

Olendo, M., Okemwa, G., Munga, C., Mulupi, L., Mwasi, L., Mohamed, H., \& Ong'anda, H. (2019). The value of long-term, community-based monitoring of marine turtle nesting: A study in the Lamu archipelago, Kenya. Oryx, 53(1), 71-80.

Pears, R. J., Choat, J. H., Mapstone, B. D., \& Begg, G. A. (2006). Demography of a Large grouper Epinephelus fuscoguttatus, from Australia's Great Barrier Reef: Implications for fishery management. Marine Ecological Progress Series, 307: 259-272.

Randall, J. E. (ed.) (1995). Coastal fishes of Oman. University of Hawaii Press, Honolulu, HI, 439 pp. 
Robinson, J., Marguerite, M., \& Isidore, M. (2007). Investigation of the importance of reef fish spawning aggregations for the sustainable management of artisanal fisheries resources in Seychelles. Edition: WIOMSA Book Series No. 6. Publisher: WIOMSA, v+42 pp.

Robinson, J., Samoilys, M. A., Grandcourt, E., Julie, D., Cedrasa, M., \& Gerry, G. (2011). The importance of targeted spawning aggregation fishing to the management of Seychelles trap fishery. Fisheries Research, 112: 96-103.

Rodrigues-Filho, J. L., Verani, J. R., Peret, A. C., Sabinson, L. M., \& Branco, J. O. (2011). The influence of population structure and reproductive aspects of the genus Stellifer (Oken, 1817) on the abundance of species on the southern Brazilian coast. Brazilian Journal of Biology, 71: 991-1002

Rosli, M. R., Ibrahim, A. L., \& Masron, T. (2014). Prediction of reef fish spawning aggregations using remote sensing: A review. IOP Conference Earth and Environmental Science Series. 18 012115. doi:10.1088/1755-1315/18/1/012115

Samoilys M. A., \& Roelofs A. (2000). Defining the reproductive biology of a Large serranid Plectropomus leopardus. CRC Reef Research Center Technical Manual No. 31, CRC Research Centre, Townsville, Australia, 36 pp.

Samoilys, M. A., Kanyange, N., Macharia, D., \& Maina, G. W. (2012). Dynamics of Siganus sutor spawning aggregations in southern Kenya.

Samoilys, M. A., Osuka, K., Mussa, J., Rosendo, S., Riddell, M., Diade, M., Mbugua, J., Kawaka, J., Hill, N., \& Koldewey, H. (2019). An integrated assessment of coastal fisheries in Mozambique for conservation planning. Ocean and Coastal Management, 182. [https://doi.org/10.1016/j.ocecoaman.2019.104924]

Santos, R. S., Silva, J. P. C., Costa, M. R., \& Araújo, F. G. (2015). O tamanho de primeira maturação como parâmetro para estabelecimento de tamanho mínimo de captura para corvina no sudeste do brasil. Boletim do Instituto de Pesca, São Paulo, 41(3): 507-518.

SCRFA (Science and Conservation of Fish Aggregations) (2018). SCRFA database. Web online database. https://www.scrfa.org/database/: SCRFA. [2013-onwards] (Acessado em 11 de Julho de 2019)

Smith, M. H., \& Heemstra, P. C. (1986). Smith's seafishes. South Africa: Springer-Verlag.

Souza-Conceição, J. M., Rodrigues-Ribeiro, M. \& Castro-Silva, M. A. (2005). Dinâmica populacional biologia reprodutiva e o ictioplâncton de Cetengraulis edentulus na enseada do Saco dos Limões, Florianópolis, Santa Catarina, Brasil. Revista Brasileira de Zoologia, 22: 953-961

Sululu, J. S., Kamukuru, A. T., Sekadende, B. C., Mahongo, S. B., \& Igulu, M. M. (2020). Reproductive biology of the anchovy (Stolephorus commersonnii, Lacepède, 1803) and spotted sardine (Amblygaster sirm, Walbaum, 1792) from Tanga Region, Tanzania. Western Indian Ocean Journal of Marine Science, 20(1): 81-94. http://dx.doi.org/10.4314/wiojms.si2020.1.8

Takemura, A., Rahman, M. S., Nakamura, S., Park, Y. J., \& Takano, K. (2004). Lunar cycles and reproductive activity in reef fishes with particular attention to Rabbitfishes. Fish and Fisheries, 5: 317-328.

Turner, S. C., Grimes, C. B., \& Able, K., W. (1983). Growth, mortality, and age/size structure of the fisheries for the tilefish, Lopholatilus chaemaelonticeps, in the middle AtlanticSouthern New England region. Fishery Bulletin of the United States, 81: 751-763 
DOI

URT (United Republic of Tanzania) \& WWF (World Wide Fund for Nature) (2005). The Status of Marine Turtles in The United Republic of Tanzania, East Africa.

Vazzoler, Anna Emília A. de M. (1996). Biologia da reprodução de peixes teleósteos: teoria e prática. Maringá: EDUEM, 169 pp.

Wambiji, N., Ohtomi, J., Fulanda, B., Kimani, E., Kullundu, N., \& Hossain, Y. (2008). Morphometric relationship and condition factor of Siganus stellatus, S. canaliculatus and S. sutor (Pisces: Siganidae) from the Western Indian Ocean waters. South Pacific Studies, 29(1): 1-15.

Woodland, D. J. (eds.) (1984). Aid to the identification of fish family Siganidae. In: FAO Species Identification Sheets for Fishery Purposes: Western Indian Ocean, Fishing Area 51, 4. Fisher W, Bianchi G (eds.), FAO, Rome.

Woodland, D. J. (1990). Revision of the fish family Siganidae with descriptions of two new species and comments on distribution and biology. Indo-Pacific Fishes, 19: 136. 